\title{
INFLUENCIA DEL CAPITAL HUMANO Y LA VIOLENCIA SOBRE EL CRECIMIENTO ECONÓMICO: PERÚ 1994-2014*
}

\author{
Recibido: 15 de mayo de 2016 • Aprobado: 11 de agosto de 2016 \\ DOI: $10.22395 /$ seec.v19n40a3 \\ Juan León Mendoza*
}

RESUMEN

El artículo tiene como objetivo conocer los efectos del capital humano y de la violencia y la inseguridad sobre el crecimiento económico departamental peruano en el período 1994-2014. La regresión econométrica efectuada con el método de los Mínimos Cuadrados Ordinarios indica la existencia de una parcial convergencia interdepartamental. La tasa de convergencia es de 0,415. La acumulación de capital humano (aproximada por los años de estudio) muestra una influencia positiva sobre el crecimiento económico departamental, en tanto que la violencia y la inseguridad (representada por los delitos contra el patrimonio) tienen un débil efecto negativo. El mayor grado de integración comercial exportadora al resto del mundo, es otra variable que también afecta de manera favorable el proceso de crecimiento económico de las diferentes regiones en Perú.

\section{PALABRAS CLAVE}

Crecimiento económico, capital humano, institucionalidad, violencia, Perú.

\section{CLASIFICACIÓN JEL}

F43, J24, K11, O43

\section{CONTENIDO}

Introducción; 1. Marco teórico y antecedentes; 2. El modelo; 3. Metodología; 4. Desempeño del Producto Bruto Interno departamental; 5. Resultados econométricos; 6. Discusión de resultados; 7. Conclusiones;

* Este artículo es una versión resumida del proyecto de investigación "Influencias del capital humano y la violencia ciudadana sobre el crecimiento económico departamental peruano" efectuado en el Instituto de Investigaciones Económicas de la Universidad Nacional Mayor de San Marcos, en el marco de los Proyectos Especiales de Investigación 2014, financiado por el Rectorado de la Universidad. El período de ejecución comprendió del mes de julio del año 2014 a diciembre del 2015.

** Economista, Universidad Nacional San Cristóbal de Huamanga, Ayacucho, Perú. Magíster en Economía, Pontificia Universidad Católica del Perú, Lima, Perú. Docente principal e investigador de la Facultad de Ciencias Económicas, Universidad Nacional Mayor de San Marcos, Lima, Perú. Dirección de correspondencia: Mz Q2 Lote 26A, Urbanización Ciudad del Pescador, Lima 02. Teléfono 996583235. Correo electrónico: jleonm@unmsm.edu.pe. 
Bibliografía.

\section{INFLUENCE OF HUMAN CAPITAL AND VIOLENCE ON ECONOMIC GROWTH: PERU 1994-2014}

\section{ABSTRACT}

The objective of this article is to learn about the effects of human capital, violence and insecurity on the economic growth of the Peruvian departments during the 1994-2014 period. The econometric regression used is based on Ordinary Least Squares Method and indicates the existence of a partial interdepartmental convergence. The convergence rate is 0,415 . The human capital accumulation (approximated for the years applicable for the study) shows a positive influence over the departmental economic growth, while violence and insecurity (represented by crimes against public heritage) shows a weak negative effect. Another variable that affects this process in a favorably is the higher level of commercial export integration.

\section{KEY WORDS}

Economic growth, human capital, institucionality, urban violence, Peru.

\section{JEL CLASSIFICATION}

F43, I24, K11, 043

\section{CONTENT}

Introduction; 1. Theoretical framework and background; 2. The model; 3. Methodology. 4. Departmental GDP performance; 5 . Econometric results; 6 . Result discussion; 7. Conclusions; Bibliography.

\section{INFLUÊNCIA DO CAPITAL HUMANO E A VIOLÊNCIA SOBRE O CRESCIMENTO ECONÓMICO: PERU 1994-2014 \\ RESUMO}

O artigo tem como objetivo conhecer os efeitos do capital humano e a violência e insegurança sobre o crescimento económico departamental peruano no período 1994-2014. A regressão econométrica efeituada com o método dos Mínimos Quadrados Ordinários indica a existência de uma parcial convergência interdepartamental. A taxa de convergência é de 0,415. A acumulação de capital humano (aproximada pelos anos de estudo) mostra uma influência positiva sobre o crescimento económico departamental, tanto que a violência e insegurança cidadã (representada pelos delitos contra o património) mostra um débil efeito negativo. O maior grau de integração comercial exportadora ao resto do mundo, é outra variável que também afeta de maneira favorável sobre dito processo de crescimento económico.

\section{PALAVRAS CHAVES}

Crescimento económico, capital humano, institucionalidade, violência cidadã, Peru

\section{CLASSIFICAÇÃO JEL}

F43, I24, K11, 043

\section{CONTEÚDO}

Introdução; 1. Marco teórico e antecedentes; 2. 0 modelo; 3. Metodologia; 4. Desempenho do Produto Bruto Interno departamental; 5 . Resultados econométricos; 6 . Discussão de resultados; 7 . Conclusões; Bibliografia. 


\section{INTRODUCCIÓN}

Después de un largo período de estancamiento, entre los años 1994-2014, el Perú experimentó un proceso de crecimiento económico más que aceptable. El producto bruto interno (PBI) creció a una tasa anual promedio de 4,66 \%. Este buen desempeño económico se produjo en un contexto en el que, por un lado, se registraron crecientes actos de violencia e inseguridad ciudadana, y por otro lado, en la literatura internacional se observa una mayor referencia al capital humano como un factor explicativo de los procesos de crecimiento económico.

En Perú no existen estudios sobre la influencia de la violencia y de la inseguridad ciudadana sobre el crecimiento económico.

En el ámbito internacional, Rubio (1995), en un estudio seminal efectuado para Colombia, encontró que la tasa de criminalidad impactó de manera negativa no solo el crecimiento económico, sino también la inversión.

Dado que la inversión en capital fijo es un factor determinante del crecimiento económico, la violencia también afecta el crecimiento mediante su efecto negativo sobre la citada inversión. En esta línea, Parra (1998) determinó que, además del capital humano, la violencia (representada por la tasa de homicidios) es un factor muy importante en la decisión de la inversión privada. Asimismo, en un estudio efectuado para México por Bernal y Castillo (2012), se halló que la ola de homicidios y secuestros tuvo efectos contractivos y significativos sobre la inversión extranjera directa.

Una investigación empírica relevante sobre una relación causal más directa entre la violencia y el crecimiento es la que efectuó Cotte (2006) para Colombia. Este halló que, además de los factores productivos tradicionales, la tasa de homicidios tuvo un impacto negativo sobre el crecimiento económico regional colombiano. Para el caso de México, González (2014) determinó econométricamente la existencia de una relación causal en sentido negativo, aunque pequeño, entre los diferentes indicadores de criminalidad y la tasa de crecimiento regional. También Robles, Calderón y Magaloni (2013) llegaron a especificar las consecuencias adversas muy significativas que genera la violencia sobre el desempeño de la actividad económica mexicana.

Referido a la influencia del capital humano sobre el crecimiento económico, se cuenta con una amplia literatura empírica en el ámbito internacional. Por ejemplo, Mankiw, Romer y Weil (1992) y Barro y Sala-i-Martín (1992) determinaron, para una muestra representativa de países, que el capital humano, medido por la tasa de escolaridad, era una de las variables que explicaban con mayor fuerza el proceso de crecimiento económico. En el mismo sentido, Barro (1996) -en una investigación que 
incluyó una muestra de 60 países- encontró que el capital humano condicionaba al crecimiento de manera decisiva.

En el marco de lo señalado se hace relevante formularse las siguientes preguntas: ¿existe un proceso de convergencia en la tasa de crecimiento de los departamentos peruanos?; ¿hasta qué punto el crecimiento económico departamental es condicionado por la acumulación del capital humano?; cla violencia y la inseguridad generan efectos negativos sobre dicho crecimiento?

En otros términos, dado que la principal fuente de acumulación de capital humano es la educación y que el delito contra el patrimonio representa el problema más importante de violencia e inseguridad ciudadana en el Perú, ¿cuál es el grado de influencia explicativa de la educación y de los delitos contra el patrimonio sobre el crecimiento económico departamental peruano?

Dadas las preguntas planteadas, en este artículo se trata de determinar la influencia del capital humano y de la violencia y la inseguridad o, en otros términos, de la educación y de los delitos contra el patrimonio, sobre el crecimiento económico departamental peruano.

Para la consecución del objetivo propuesto, con información estadística que corresponde a los 24 departamentos peruanos y para el período 1994-2014, se efectúan regresiones econométricas mediante el uso del método de los Mínimos Cuadrados Ordinarios (OLS).

El presente artículo adquiere una singular importancia debido a la carencia de investigaciones puntuales sobre el crecimiento económico peruano con información que involucre a todos los departamentos y que incluya el capital humano, y la violencia y la inseguridad como variables explicativas.

Los resultados del estudio indican que la acumulación de capital humano y el mayor grado de integración comercial exportadora al resto del mundo son las variables más importantes que determinan de manera positiva la evolución de la producción departamental peruana en el largo plazo, en tanto que el problema de la violencia y la inseguridad ciudadana tiene un rol negativo, pero, de mucha menor importancia.

El artículo se divide en siete secciones. En la primera, se desarrolla el marco teórico relevante y se sistematizan los pocos trabajos empíricos efectuados sobre el crecimiento de algunos departamentos peruanos; en la segunda sección, se formula un modelo económico donde se introducen de manera formal las variables explicativas del capital humano y la violencia ciudadana; en la tercera, se desarro- 
llan los aspectos metodológicos; en la cuarta, se expone la evolución de la tasa de crecimiento del producto bruto interno departamental; en la quinta, se muestran los resultados que arrojan las regresiones econométricas efectuadas; en la sexta, se discuten los resultados hallados, y en la última sección, se presentan las conclusiones.

\section{MARCO TEÓRICO Y ANTECEDENTES}

Solow (1957) formuló un modelo que mostraba que la tasa de crecimiento de la producción, en el largo plazo, dependía del ritmo de acumulación del capital físico, de la tasa de crecimiento de la mano de obra y del "residuo de Solow". Dicho residuo incluye el efecto del progreso tecnológico, el cual se considera exógeno.

A partir de la década de 1980 se empezaron a formular los denominados modelos de crecimiento económico endógeno.

En esta línea, para Romer (1994) y Lucas (1988), los procesos de investigación e innovación explicaban el cambio tecnológico; a su vez, dicho cambio tecnológico estaba asociado a la acumulación de habilidades y conocimientos humanos, es decir, a la acumulación de capital humano. Así, el capital humano se convertía en una de las principales variables que determinaban el crecimiento económico.

A la par con el desarrollo de la teoría, en el plano empírico de la sociedad contemporánea se tiende a aceptar la creciente importancia del capital humano en el proceso productivo. Cada vez se observa una mayor presencia de sectores económicos intensivos en conocimientos y habilidades humanas, de modo que se entra en lo que podemos llamar la era de una sociedad del conocimiento.

Las teorías de crecimiento endógeno ponen énfasis en el capital humano como uno de los factores explicativos más importantes del crecimiento económico. Sin embargo, North (1993) sostiene que el desarrollo institucional es también un factor decisivo.

Por desarrollo institucional, se entiende a la presencia de reglas y normas formales e informales que posibilitan la plena vigencia de los estados de derecho y el respeto de la propiedad privada; de este modo, en un escenario de plena institucionalidad (reglas claras, estables y óptimas) los recursos son asignados de manera eficiente.

En ese sentido, Rodrick, Subramanian y Trebbi (2002) sostienen que cuando rige la institucionalidad necesaria, se crean condiciones favorables para la inversión productiva, por ende, se posibilitan mayores tasas de crecimiento en la producción. 
La expresión de una baja institucionalidad, es decir, de la vigencia parcial del estado de derecho, lo representa la violencia, además de la inseguridad ciudadana asociada a los robos, asaltos, asesinatos o crímenes. Dichos fenómenos generan inseguridad e inestabilidad, de modo que afectan las inversiones de forma negativa.

Según Hofstetter (1998), la inclusión del fenómeno de la violencia en los modelos de crecimiento neoclásico muestra con claridad la forma en que este desacelera el crecimiento tanto en el corto como en el largo plazo. La violencia afecta de manera negativa el proceso de acumulación del capital físico y humano (inversión en capital físico y humano), y por tanto, las productividades factoriales. A su vez, la violencia perjudica el crecimiento económico debido a que en muchos casos ello implica la destrucción de la infraestructura física, la pérdida de vidas humanas y lesiones, entre otros.

En Perú, un trabajo preliminar referido al efecto de la violencia ciudadana sobre el crecimiento económico es el efectuado por León (2005). El autor muestra que los delitos contra la libertad condicionaron, de alguna manera y en forma negativa, el proceso de crecimiento en el período 1990-2003.

Se cuenta con pocas investigaciones que han tratado de identificar las barreras para el crecimiento económico de los departamentos peruanos. Así, se tiene el trabajo efectuado por Armendáriz, Jaramillo y Zegarra (2011) para el caso del departamento de Junín; de Webb, Mendieta y Agreda (2012) para Apurímac; y el de Mendoza y Gallardo (2011) para Cajamarca.

Dichos autores hallaron que la baja dotación de capital humano, la mala infraestructura física y el alto costo del crédito para financiar las inversiones eran los principales elementos comunes que limitaban el crecimiento de los departamentos analizados.

A continuación, se formula un modelo económico de crecimiento que incluye el capital humano, y la violencia y la inseguridad como variables explicativas.

\section{EL MODELO}

En la línea de lo propuesto por Solow (1957) se asume que el crecimiento económico está asociado con la acumulación del capital físico o inversión, financiada por el ingreso nacional no consumido, es decir, por el ahorro, el mismo que es una proporción $s$ del ingreso nacional.

El producto bruto interno (Y), en términos tecnológicos, depende del uso del factor capital físico (K), del factor trabajo (L) y del capital institucional (X). El capital 
institucional genera externalidades sobre la productividad de los factores. Por ello, en forma similar que el modelo con externalidades desarrollado por Romer (1989), se formula la siguiente función de producción Cobb-Douglas con presencia de externalidades del capital institucional:

$$
\mathrm{Y}=\mathrm{AK}^{\propto} \mathrm{L}^{1-\alpha} \mathrm{X}^{\beta}
$$

Donde, A es la variable tecnología y $\beta$, un parámetro que capta el grado de eficacia de la institucionalidad sobre el capital físico y el factor trabajo.

La inversión bruta (I) es igual a la variación del stock de capital físico en el tiempo $(\hat{K})$ más el monto de la depreciación del capital $(d K)$. Donde $d$ es la tasa de depreciación:

$$
\mathrm{I}=\hat{\mathrm{K}}+d \mathrm{~K}
$$

Si se efectúan los reemplazos correspondientes, se deriva la siguiente ecuación de acumulación del capital físico en el tiempo:

$$
\hat{\mathrm{K}}=s A K^{\propto} \mathrm{L}^{1-\alpha} \mathrm{X}^{\beta}-d \mathrm{~K}
$$

Si se divide la ecuación [3] por el factor trabajo, se tiene:

$$
\frac{\hat{\mathrm{K}}}{\mathrm{L}}=s \mathrm{~A} k^{\alpha} \mathrm{X}^{\beta}-d k
$$

Dado:

$$
\hat{k}=\frac{\hat{K}}{\mathrm{~L}}-k n
$$

Donde, $n$ es la tasa de crecimiento de la población y $k$, el capital físico per cápita.

Al reemplazar [4] en [5], se deriva la ecuación de acumulación del capital físico per cápita en el tiempo:

$$
\hat{k}=s A k^{\alpha} X^{\beta}-(n+d) k
$$

Bajo el supuesto de $\hat{k}=0$, se halla el valor de la producción per cápita en situación de equilibrio estacionario $\left(y^{*}\right)$ :

$$
y^{*}=\left[\frac{s A}{n+d}\right]^{\frac{\alpha}{1-\alpha}} X^{\frac{\beta}{1-\alpha}}
$$


La ecuación [7] indica que la variable institucional X tiene un efecto positivo sobre el nivel de la producción per cápita de largo plazo: a mayor institucionalidad, el valor de la producción per cápita también será mayor.

Si se divide [6] por el capital per cápita, y bajo el supuesto de que $\frac{\hat{y}}{y}=\propto \frac{\hat{k}}{k}$, se obtiene la ecuación de la tasa de crecimiento del producto per cápita en el tiempo:

$$
\frac{\hat{y}}{y}=\propto\left(\frac{s A X^{\beta}}{k^{1-\alpha}}-(n+d)\right)
$$

Según la ecuación [8], la tasa de crecimiento del producto per cápita también está condicionada, en sentido directo, por factores de corte institucional (X). La producción per cápita crecerá en el tiempo en la medida en que $\left(\frac{s A X^{\beta}}{k^{1-\alpha}}\right)$ sea mayor que la suma de la tasa de crecimiento de la población y la tasa de depreciación $(n+d)$.

La presencia de la externalidad institucional afecta de manera positiva la tasa de crecimiento del producto per cápita. Sin embargo, si en un momento inicial hubiera crecimiento económico, ello será transitorio en tanto que no se alcance la situación de equilibrio estacionario. En el tiempo, por la presencia de la productividad marginal decreciente del capital, la tasa de crecimiento del capital per cápita y del producto per cápita tenderá a disminuir hasta llegar a cero en la situación de equilibrio estacionario de largo plazo. Así, el modelo formulado indica que el desarrollo institucional afectará al crecimiento económico solo de forma transitoria y no de manera permanente.

¿Cuál es la relación entre el capital humano y la violencia con el capital institucional?

En principio, Becker (1993) sostiene que la acumulación del capital humano (stock de conocimientos y habilidades humanas) depende de manera muy importante de la educación.

La educación coadyuva a elevar el nivel de información que manejan los individuos tal que genera conductas económicas más eficientes y externalidades positivas sobre otros, lo que afecta para bien a la institucionalidad en la sociedad. Además, la educación eleva la capacidad de los individuos de respetar las reglas institucionales establecidas. De ahí que Glaeser y otros (2004) asumen que el capital humano y la educación influyen de manera positiva sobre los aspectos institucionales. En la misma dirección, según McMahon (2000), las externalidades positivas de la educa- 
ción coadyuvan al desarrollo institucional a través de sus efectos favorables sobre la estabilidad política, el respeto a los derechos humanos y la democracia.

Una alta institucionalidad se manifiesta en la plena vigencia del Estado de derecho y el respeto irrestricto de la propiedad privada. Por ello, los actos delictivos en general significan un retroceso institucional. En ese sentido, Tudela y López (2005) sostienen que la delincuencia, expresada en actos de violencia, en tanto que afecta a la seguridad ciudadana y colectiva, perjudica el desarrollo institucional.

De lo expuesto, se asume que el capital institucional (X) está en función del capital humano $(\mathrm{H})$, de la vigencia del Estado de derecho relacionada con la violencia y la inseguridad (V), y de otros factores diferentes al capital humano y la violencia $(\mathrm{O})$ :

$$
\mathrm{X}=f(\mathrm{H}, \mathrm{V}, \mathrm{O})
$$

Al efectuar el reemplazo de [9] en [8] se tiene:

$$
\frac{\hat{y}}{y}=\propto\left(\frac{\operatorname{sAf}(\mathrm{H}, \mathrm{V}, \mathrm{O})^{\beta}}{k^{1-\alpha}}-(n+d)\right)
$$

Según la ecuación [10], la tasa de crecimiento del producto per cápita depende, en sentido directo, de la acumulación de capital humano, y en sentido inverso, de la violencia. A mayor stock de capital humano y menor violencia, se tendrá una mayor tasa de crecimiento económico. En otros términos, en la medida en que los individuos alcancen mayores niveles de educación formal y que haya menor violencia e inseguridad, la producción per cápita mostrará tendencias al crecimiento.

\section{METODOLOGÍA}

Para efectos de la regresión estadística a ejecutar, primero, se expresa el modelo económico formulado en términos de un modelo econométrico. En dicho modelo econométrico, la variable explicativa del capital humano se aproxima mediante la variable educación, debido a que según Becker (1993), la educación es el factor más importante que determina la acumulación de capital humano. A su vez, la variable educativa se cuantifica mediante los años de estudio alcanzados por la población.

La variable violencia e inseguridad se representa a través de los delitos contra el patrimonio (hurto, robo, estafas, apropiación ilícita y fraude), debido a que es el tipo de delito más recurrente en el Perú, con una participación cercana al 70 \% del total de delitos registrados. 
El segundo tipo de delito más frecuente es el delito contra la vida, el cuerpo y la salud (homicidios y lesiones), aunque con una participación más pequeña. Este también se incorpora en la regresión como otra variable explicativa relacionada con la violencia y la inseguridad.

El crecimiento de la producción no depende solo del capital humano y la violencia. La literatura empírica suele considerar otras variables explicativas. Por ello, en el sentido de lo trabajado por Barro (1991), Thirlwall (2003) y Querubín (2003), en el modelo econométrico también se incluyen: la inversión en capital físico, el grado de apertura comercial, las regiones geográficas, y la desigualdad en la distribución de ingresos.

Entonces, se regresiona la siguiente ecuación econométrica:

$$
\mathrm{Y}_{i}=\beta_{0}+\beta_{1} \cdot \mathrm{Y}_{0 i}+\beta_{2} \cdot \mathrm{X}_{2 i}+\beta_{3} \cdot \mathrm{X}_{3 i}+\beta_{4} \cdot \mathrm{X}_{4 i}+\beta_{5} \cdot \mathrm{X}_{5 i}+\beta_{6} \cdot \mathrm{X}_{6 i}+\beta_{7} \cdot \mathrm{X}_{7 i}+\beta_{8} \cdot \mathrm{X}_{8 i}+u
$$

\section{Variable dependiente:}

$\mathrm{Y}_{i}=\mathrm{TCPBI}=$ Tasadecrecimiento del $\mathrm{PBI}$ per cápita del departamento $\mathrm{i}$

\section{Variables independientes:}

$$
\begin{aligned}
& \mathrm{Y}_{o \mathrm{i}}=\mathrm{LPBII}=\text { Logaritmodel PBI per cápitainicial del departamento } i \\
& \mathrm{X}_{2 i}=\mathrm{TDCVCS}=\text { Tasa de delitos contrala vida, el cuerpo y la saluddel dpto. } i \\
& \mathrm{X}_{3 i}=\mathrm{TDCPATRI}=\text { Tasa de delitos contra el patrimonio del departamento } i \\
& \mathrm{X}_{4 i}=\mathrm{REGION}=\text { Región geográfica alaque pertenece el departamento } i \\
& \mathrm{X}_{5 i}=\mathrm{INV}=\text { Tasadeinversióndel departamento } i \\
& \mathrm{X}_{6 i}=\text { DESIGUAL }=\text { Desigualdadenladistribucióndeingresos del dpto. } i \\
& \mathrm{X}_{7 i}=\mathrm{APERTU}=\text { Gradode apertura ointegración comercial del departamento } i \\
& \mathrm{X}_{8 \mathrm{i}}=\mathrm{AÑ} O \mathrm{EST}=\mathrm{A} \tilde{n} o s \text { de estudio alcanzados por la población del departamento } \mathrm{i}
\end{aligned}
$$

La inclusión del PBI del período inicial para cada departamento permite determinar si hay o no convergencia en el proceso de crecimiento económico departamental. Se incorpora dicha variable en términos de logaritmo para así poder estimar el valor de la tasa de convergencia de manera directa. 
Tanto la tasa de delitos contra la vida, el cuerpo y la salud como la tasa de delitos contra el patrimonio se definen y cuantifican como la cantidad de cada tipo de delito registrado por cada mil habitantes para cada departamento.

Se incorpora la variable región, a fin de evaluar si la ubicación geográfica del departamento condiciona su proceso de crecimiento. Esta es una variable dicotómica que toma el valor de cero para aquellos ubicados en la sierra y la selva, y de uno para aquellos localizados en la región de la costa. Se asume que los de la costa poseen mayores facilidades para el crecimiento debido a que limitan con el litoral marítimo y tienen acceso directo a los puertos.

La tasa de inversión es igual al valor de la inversión en infraestructura dividido entre el PBI. La desigualdad en la distribución de ingresos se representa con el coeficiente de Gini. El grado de integración comercial se calcula mediante la división de la exportación entre el producto bruto interno.

Se espera encontrar los siguientes signos:

$$
\beta_{2}<0, \beta_{3}<0, \beta_{4}>0, \beta_{5}>0, \beta_{6}>0 \text { ó } \beta_{6}<0, \beta_{7}>0, \beta_{8}>0
$$

Para efectos de la regresión, se utiliza el método de los Mínimos Cuadrados Ordinarios, el paquete estadístico Eviews 6 y la información correspondiente a los 24 departamentos peruanos para el período 1994-2014. La Provincia Constitucional del Callao queda incluida dentro del departamento de Lima'

El período analizado abarca los años 1994-2014 por las siguientes razones: primero, porque en el período precedente (1987-1993) hubo años de gran inestabilidad y crisis económica de corto plazo; segundo, debido que a partir del año 1994, después de las políticas de estabilización económica y reformas radicales implementadas en los años 1990-1993, la economía peruana recién entró a una etapa de estabilidad y crecimiento.

La regresión se ejecuta con variables expresadas en términos de valores promedio que corresponden a los años 1994 a 2001 y 2002 a 2014. Los datos se dividen en esos dos períodos porque, por un lado, el crecimiento económico fue lento en el primer período y mucho más rápido en el segundo, y por otro lado, porque ello permite incrementar el número de observaciones de 24 a 48.

Se utilizan valores promedio de las variables de cada período debido a que no se cuenta con la información anual completa para todo el período de análisis. Por ejemplo, no existe información para algunos años sobre el número de delitos, la inversión y las exportaciones departamentales.

${ }^{1}$ El lector que desee acceder a la base de datos utilizada en las regresiones puede solicitarlo al siguiente correo: juanleon12000@yahoo.es. 
Con la finalidad de determinar si hubo o no convergencia en la tasa de crecimiento departamental, se considera el producto bruto interno de los años 1994 y 2002 como la producción del año inicial.

La información sobre el PBI departamental se recabó del Instituto Nacional de Estadística e Informática (INEI 2003, 2013 y 2015).

En cuanto se refiere a la variable delitos contra el patrimonio y delitos contra la vida, el cuerpo y la salud, la fuente estadística utilizada fue el Compendio Estadístico del Perú, el mismo que también es publicado por el INEI (2015a) desde el año 1983.

Dicho compendio, en su capítulo de Seguridad y Orden Público, contiene información sobre los diferentes tipos de delitos ocurridos en cada departamento peruano, para la mayoría de los años del período de análisis.

Respecto a las demás variables explicativas, complementario a la información proveniente del Compendio Estadístico del Perú, se tomaron como fuentes: la Asociación de Exportadores (ADEX, 2015) para el caso de las exportaciones, el INEI (2014) para los años de estudio de la población, y Ponce (2013) para la inversión y el coeficiente de Gini.

\section{DESEMPEÑO DEL PRODUCTO BRUTO INTERNO DEPARTAMENTAL}

En el período de análisis, en el marco de un moderado crecimiento económico nacional, se registraron grandes diferencias en las tasas de crecimiento departamentales: las velocidades del crecimiento no fueron homogéneas (véase tabla 1).

Tabla 1. Perú: tasa de crecimiento del producto bruto interno departamental, 1994-2014

\begin{tabular}{|l|c|c|}
\hline \multicolumn{1}{|c|}{ Departamentos } & Tasa de crecimiento acumulada (\%) & Tasa de crecimiento promedio anual (\%) \\
\hline Amazonas & 206,45 & 6,72 \\
\hline Ancash & 142,86 & 5,48 \\
\hline Apurímac & 96,67 & 4,28 \\
\hline Arequipa & 159,49 & 5,52 \\
\hline Ayacucho & 163,68 & 5,65 \\
\hline Cajamarca & 147,58 & 5,30 \\
\hline Cusco & 156,86 & 5,62 \\
\hline Huancavelica & 50,75 & 2,35 \\
\hline Huánuco & 78,56 & 3,49 \\
\hline Ica & 171,01 & 5,86 \\
\hline
\end{tabular}


Influencia del capital humano y la violencia sobre el crecimiento económico: Perú 1994-2014

\begin{tabular}{|l|c|c|}
\hline \multicolumn{1}{|c|}{ Departamentos } & Tasa de crecimiento acumulada (\%) & Tasa de crecimiento promedio anual (\%) \\
\hline Junín & 102,77 & 4,08 \\
\hline La Libertad & 161,18 & 5,57 \\
\hline Lambayeque & 98,06 & 4,01 \\
\hline Lima & 156,31 & 5,43 \\
\hline Loreto & 81,47 & 3,45 \\
\hline Madre de Dios & 92,89 & 4,05 \\
\hline Moquegua & 84,60 & 3,61 \\
\hline Pasco & 84,60 & 3,61 \\
\hline Piura & 108,32 & 4,23 \\
\hline Puno & 113,72 & 4,34 \\
\hline San Martín & 124,68 & 4,82 \\
\hline Tacna & 121,49 & 4,62 \\
\hline Tumbes & 83,90 & 3,82 \\
\hline Ucayali & 173,14 & 5,88 \\
\hline
\end{tabular}

Fuente: Elaboración propia con información del INEI (2003, 2013 y 2015).

Por ejemplo, las tasas de crecimiento acumuladas de los departamentos de Amazonas y Ucayali fueron de 206,5 \% y $173,1 \%$, respectivamente, en tanto que los departamentos de Huancavelica y Huánuco alcanzaron tasas mucho más bajas de $50,8 \%$ y $78,6 \%$, respectivamente.

Una lectura de la tabla 1 muestra que, entre los ocho primeros departamentos con la mayor tasa de crecimiento figuran cuatro que están ubicados totalmente en las regiones de la sierra y la selva (Amazonas, Ucayali, Ayacucho y Cuzco), y dos en la costa marítima con una mayor proporción de su geografía localizada en la sierra (La Libertad y Arequipa). En tanto que entre los ocho departamentos con la menor tasa de crecimiento predominan aquellos que están localizados en las regiones de la sierra y la selva (Huancavelica, Huánuco, Loreto, Pasco, Madre de Dios y Apurímac).

Dado que los departamentos más pobres y de menores ingresos del Perú están localizados en las regiones de la sierra y la selva, el hecho de que entre los de mayor crecimiento económico figuren algunos que están ubicados en estas regiones y que entre los de menor crecimiento también predominen departamentos pertenecientes a estas mismas regiones, indica hasta cierto punto, que en el Perú se tiene una parcial convergencia en el proceso de crecimiento departamental. 


\section{RESULTADOS ECONOMÉTRICOS}

La tabla 2 muestra el resultado de una primera regresión efectuada con todas las variables explicativas del modelo econométrico especificado en la sección 3.

Todos los parámetros estimados tienen el signo esperado. Solo algunos de ellos presentan significancia estadística. Las variables años de estudio (AÑOEST), el grado de apertura comercial (APERTU) y el logaritmo del PBI del período inicial (LPBII) muestran significancia estadística al $1 \%$ de error. La variable tasa de delitos contra el patrimonio (TDCPATRI), si bien no es estadísticamente significativa al $1 \%$ de error, presenta una mayor significancia estadística entre las variables no significativas.

\section{Tabla 2. Resultado de la regresión inicial}

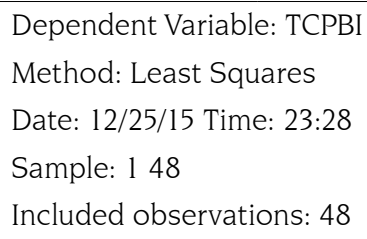

$\begin{array}{ccccc}\text { Variable } & \text { Coefficient } & \text { Std. Error } & t \text {-Statistic } & \text { Prob. } \\ \text { LPBII } & -0,409095 & 0,803448 & -5,091755 & 0,0000 \\ \text { TDCVCS } & -0,255604 & 0,313753 & -0,814667 & 0,4202 \\ \text { TDCPATRI } & -0,233194 & 0,188272 & -1,238602 & 0,2229 \\ \text { REGION } & 0,165167 & 0,810710 & 0,203731 & 0,8396 \\ \text { INV } & 0,085309 & 0,124863 & 0,683217 & 0,4985 \\ \text { DESIGUAL } & 0,017788 & 0,060241 & 0,295278 & 0,7693 \\ \text { APERTU } & 0,048086 & 0,012596 & 3,817513 & 0,0005 \\ \text { ANOEST } & 1,654595 & 0,223039 & 7,418394 & 0,0000 \\ \text { C } & 21,16682 & 5,986869 & 3,535541 & 0,0011\end{array}$

R-squared $\quad 0,651612$

Mean dependent var

2,362708

Adjusted R-

squared $\quad 0,580147$

S,D, dependent var

2,539902

S.E. of regression

1,645756

Akaike info criterion

4,001637

Sum squared resid

105,6319

Schwarz criterion

4,352487

Log likelihood

$-87,03928$

Hannan-Quinn criter,

4,134224

F-statistic

9,118008

Durbin-Watson stat

2,155377

Prob(F-statistic) $\quad 0,000001$

Fuente: Estimación propia con el programa Eviews 6. 
Después de excluir las variables sin significancia estadística (apoyado en el Test estadístico de Variables Redundantes), se efectúa una regresión final.

En la regresión final expuesta en la tabla 3, se observa que todas las variables explicativas consideradas son estadísticamente significativas. El logaritmo del PBI inicial, la apertura comercial y los años de educación son estadísticamente significativos al $1 \%$ de margen de error, en tanto que la tasa de delitos contra el patrimonio también lo es, pero, con un menor grado de confianza (10 \% de error).

El resultado de la regresión final señala que el capital humano, representado por los años de educación, condiciona de forma positiva el proceso de crecimiento económico departamental en el Perú, en tanto que la violencia ciudadana, representado por los delitos contra el patrimonio, lo hace de manera negativa y débil. El grado de integración comercial de los departamentos a la economía internacional también influye de modo positivo sobre dicho crecimiento.

\section{Tabla 3. Resultado de la regresión final}

\begin{tabular}{|c|c|c|c|c|}
\hline \multicolumn{5}{|c|}{ Dependent Variable: TCPBI } \\
\hline \multicolumn{5}{|c|}{ Method: Least Squares } \\
\hline \multicolumn{5}{|c|}{ Date: $12 / 25 / 15$ Time: $23: 31$} \\
\hline \multicolumn{5}{|c|}{ Sample: 148} \\
\hline \multicolumn{5}{|c|}{ Included observations: 48} \\
\hline Variable & Coefficient & Std, Error & t-Statistic & Prob, \\
\hline LPBII & $-0,414814$ & 0,760507 & $-5,454446$ & 0,0000 \\
\hline TDCPATRI & $-0,256464$ & 0,136002 & $-1,885730$ & 0,0661 \\
\hline APERTU & 0,047412 & 0,011269 & 4,207250 & 0,0001 \\
\hline ANOEST & 1,655964 & 0,206311 & 8,026535 & 0,0000 \\
\hline C & 22,55247 & 5,170179 & 4,362028 & 0,0001 \\
\hline R-squared & 0,642159 & \multicolumn{2}{|c|}{ Mean dependent var } & 2,362708 \\
\hline $\begin{array}{l}\text { Adjusted R- } \\
\text { squared }\end{array}$ & 0,608871 & \multicolumn{2}{|c|}{ S,D, dependent var } & 2,539902 \\
\hline $\mathrm{S}, \mathrm{E}$, of regression & 1,588461 & \multicolumn{2}{|c|}{ Akaike info criterion } & 3,861741 \\
\hline $\begin{array}{c}\text { Sum squared } \\
\text { resid }\end{array}$ & 108,4980 & \multicolumn{2}{|c|}{ Schwarz criterion } & 4,056658 \\
\hline Log likelihood & $-87,68179$ & \multicolumn{2}{|c|}{ Hannan-Quinn criter, } & 3,935401 \\
\hline F-statistic & 19,29128 & \multicolumn{2}{|c|}{ Durbin-Watson stat } & 2,116273 \\
\hline Prob(F-statistic) & 0,000000 & & & \\
\hline
\end{tabular}

Fuente: Estimación propia con el programa Eviews 6. 
La regresión indica que existe convergencia condicional en el proceso de crecimiento de los departamentos peruanos: en promedio, los de menor ingreso crecen a una tasa mayor que los de mayor ingreso. La tasa de convergencia es de 0,415.

La muestra analizada no es muy grande (48 observaciones) y es menor que 100, por lo que se hace necesario efectuar el Test de Normalidad de los Residuos. Para tal fin se utiliza el criterio del estadístico de la prueba Jarque-Bera. El resultado es igual a

$$
\mathrm{X}_{(4) 0.05}^{2}=9,49 \text { Jarque-Bera }=0,499988 .
$$

Debido a que 0,499988 es menor que 9,49, se acepta la hipótesis nula de que los residuos siguen una distribución normal, de modo tal que se cuenta con la justificación teórica correspondiente para que sean utilizados en las pruebas estadísticas que involucren las distribuciones de $\mathrm{t}$ y $\mathrm{F}$.

El test gráfico de estabilidad de parámetros (Test de CUSUM al cuadrado), a un $5 \%$ de margen de error, muestra que los parámetros estimados del modelo son estables, de manera que son confiables para fines de inferencia y pronóstico. Como se puede observar en el gráfico 1, la línea ondulante, que representa la evolución del valor de los parámetros no sobrepasa la franja lineal.

\section{Gráfico 1. Estabilidad de los parámetros}

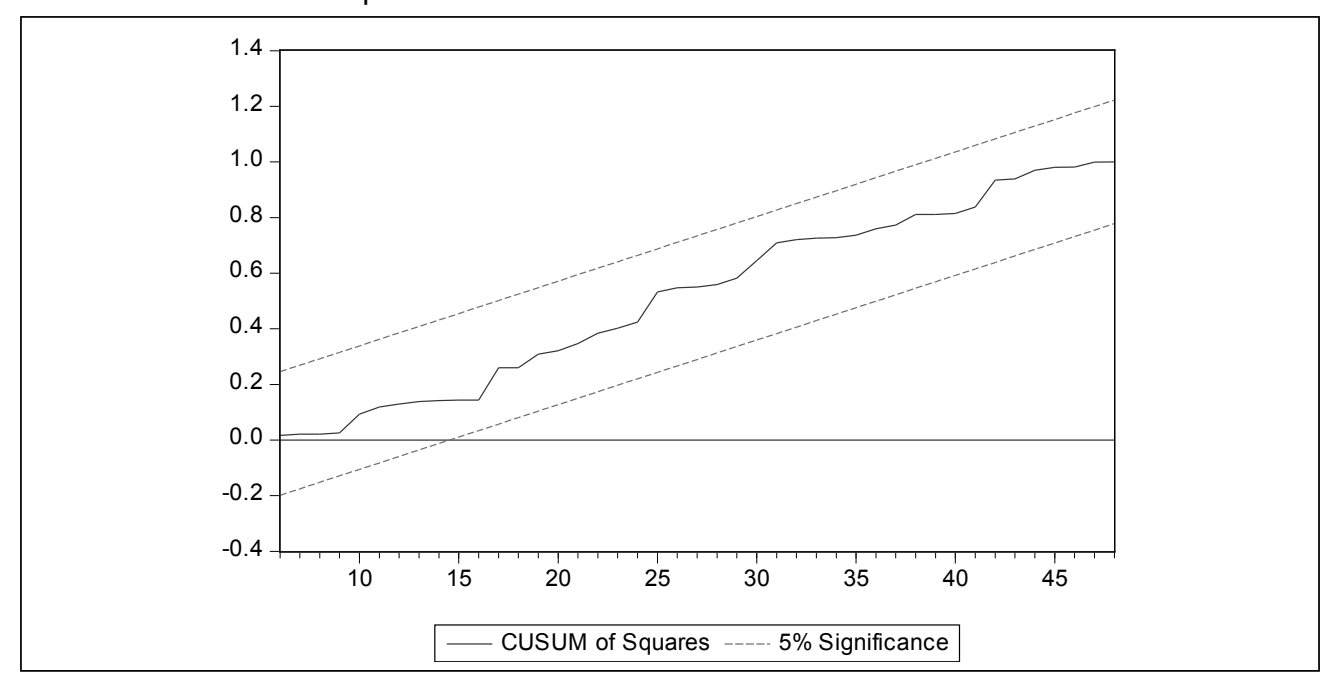

Fuente: Elaboración propia

El ajuste del modelo estimado es bueno. Como se puede visualizar en el gráfico 2, la línea del modelo estimado se ajusta muy bien a la línea de los datos observados.

En la parte inferior del gráfico 2, se observa que la línea del diagrama de los residuos fluctúa de manera errática o aleatoria alrededor de cero, tal que los residuos 
presentan un comportamiento aleatorio, no sistemático o irregular. Este resultado ratifica la hipótesis nula de que no existe autocorrelación de errores.

Dada el ajuste del modelo final, y de que éste cumple con los supuestos establecidos de una regresión lineal, se puede afirmar que el modelo puede explicar de forma eficiente el comportamiento de la variable estudiada, es decir, de la tasa de crecimiento económico departamental peruano.

Gráfico 2. Bondad de ajuste del modelo estimado

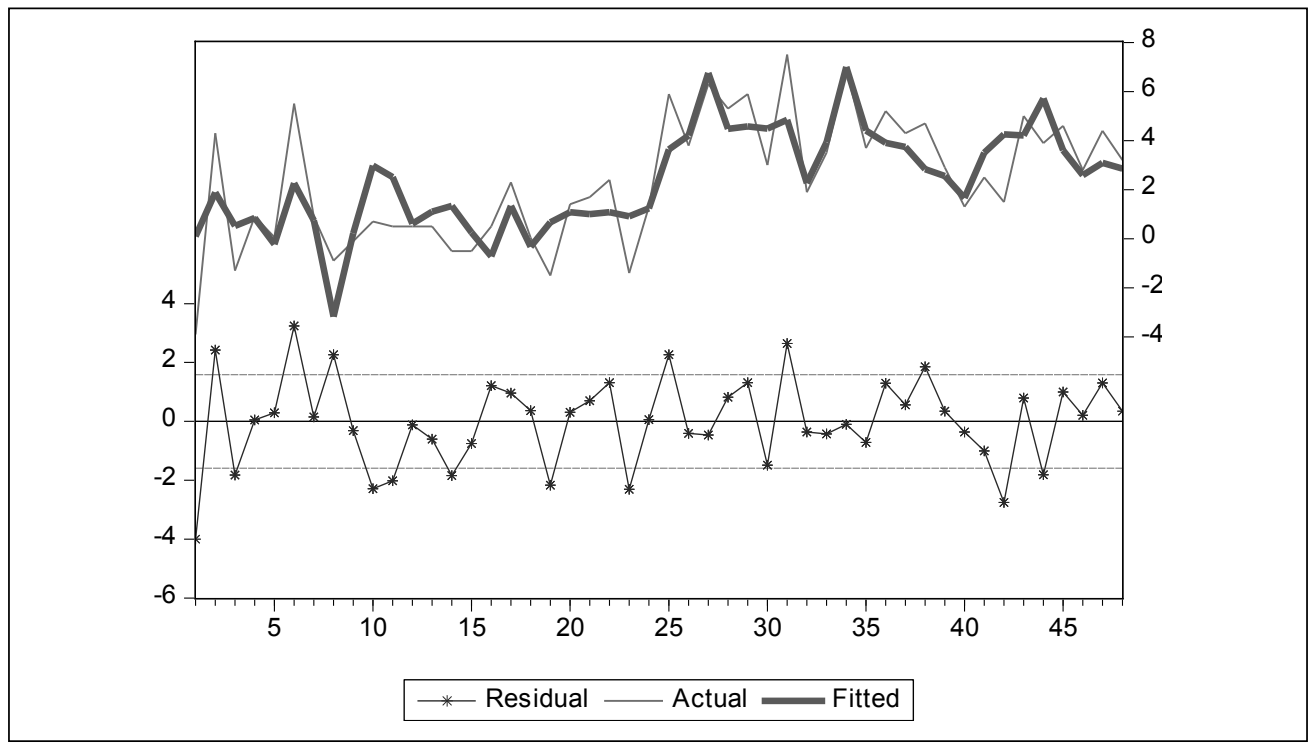

Fuente: elaboración propia

\section{DISCUSIÓN DE RESULTADOS}

En Perú, se tiene un proceso de convergencia: en promedio, los departamentos de menores ingresos crecen más rápido que aquellos de mayores ingresos. La tasa de convergencia es de 0,415.

No obstante, el proceso de convergencia no es generalizado, sino parcial. Es decir, hay algunos departamentos de menor ingreso que crecen a tasas mayores que el promedio, pero también existen otros - con un ingreso similar-que lo hacen a tasas por debajo del promedio.

Por ejemplo, en el período de análisis, entre los primeros cuatro departamentos con la mayor tasa de crecimiento, figuran tres que están entre los de menor ingreso y ubicados en las regiones de la sierra y selva (Amazonas, Ucayali y Ayacucho). Pero, entre los cuatro departamentos con la más baja tasa de crecimiento, también figuran 
tres que son de menor ingreso y que pertenecen a las mismas regiones (Huancavelica, Loreto y Huánuco). Ello indica que en un proceso de convergencia el primer grupo de los departamentos citados está involucrado, mas no así los segundos.

Según el Instituto Nacional de Estadística e Informática, relacionado con el problema y la violencia e inseguridad en el Perú, el tipo de delito predominante es el delito contra el patrimonio, con una tendencia a un gran incremento a partir del año 2008.

En el marco del resultado hallado en la regresión, se podría afirmar que la violencia y la inseguridad ciudadana, asociada a los delitos contra el patrimonio, implica un obstáculo parcial para el crecimiento económico peruano. Es decir, los robos, los hurtos, las estafas y los fraudes perjudican, aunque de manera débil, el proceso de crecimiento departamental.

En el mismo sentido de los resultados hallados por Armendáriz, Jaramillo y Zegarra (2011) para el departamento de Junín, de Webb, Mendieta y Agreda (2012) para Apurímac, de Mendoza y Gallardo (2011) para Cajamarca, el presente estudio ha determinado que el capital humano es un factor que condiciona de manera muy importante el crecimiento económico de los departamentos peruanos. Los departamentos que más han crecido son aquellos que poseen los mayores niveles de capital humano, es decir, aquellos que cuentan con una población con mayores años de estudio.

En efecto, en la denominada era o sociedad del conocimiento, el capital humano (stock de conocimientos y habilidades humanas) se ha revalorizado, de manera tal que se ha convertido en un factor decisivo y condicionante no solo del crecimiento económico, sino también de muchos hechos económicos, sociales y políticos.

En la gestión empresarial y gubernamental en general, la dotación de personas con las mayores habilidades y conocimientos ha cobrado mayor importancia. Inclusive, en el proceso de la identificación, formulación y ejecución de los proyectos de inversión, el capital humano se ha traducido en un factor más relevante que el capital financiero y físico. En el mismo sentido, en el ámbito nacional e internacional, el comercio de bienes y servicios se ha vuelto también cada vez más intensivo en conocimientos.

Entonces, en tanto que las variables educativas determinan la acumulación del capital humano, la educación se ha tornado en un factor de máxima importancia para el crecimiento económico. De ahí que no causa sorpresa alguna que, en la regresión efectuada, la variable años de estudio de la población muestre significancia estadística. 
Thirlwall (2003) señala que la apertura y la integración comercial afectan de modo positivo el crecimiento económico, porque presionan a las empresas y a las instituciones a ser más eficientes. A su vez, permiten el acceso a nuevas tecnologías, conocimientos y variedad de bienes, lo cual impacta de manera favorable en la productividad. Además, para un país con un mercado interno pequeño, la integración comercial genera oportunidades de acceso a mercados externos mucho más grandes o enormes, de modo que ello coadyuva a la generación de economías de escala, a la mayor producción y exportación, y por ende, a un incremento en la tasa de crecimiento económico.

Por ello, la mayor apertura e integración comercial que ha establecido el Perú en el marco de los tratados de libre comercio (TLC) habría impactado de modo favorable el desempeño económico de los departamentos peruanos, es decir, los departamentos peruanos más integrados y abiertos al comercio internacional, de alguna manera habrían elevado su competitividad y productividad, por lo que han alcanzado mayores tasas de crecimiento económico.

\section{CONCLUSIONES}

Existe un proceso parcial de convergencia en el crecimiento de los diferentes departamentos peruanos: algunos departamentos de menores ingresos tienden a crecer a tasas mayores que aquellos con mayores ingresos. La tasa de convergencia es cercana a 0,415 .

La regresión econométrica efectuada indica que el capital humano es uno de los principales factores que explican el crecimiento económico departamental en el Perú. Entonces, los departamentos que más crecen son aquellos que poseen una población con una mayor cantidad promedio de años de estudio.

Otro factor que explica el crecimiento económico departamental es el grado de integración comercial al resto del mundo. Los departamentos que más crecen son los que están más conectados al exterior, es decir, aquellos que destinan una mayor proporción de su producción al mercado internacional.

La violencia y la inseguridad, aproximada por la tasa de delitos contra el patrimonio, tiene una influencia negativa, pero débil. Este hecho significa que la alta tasa de delitos contra el patrimonio que se observa, no ha representado un gran obstáculo para el proceso de crecimiento económico de los departamentos peruanos.

La baja significancia estadística de los delitos contra el patrimonio podría ser reflejo del problema de la calidad y cantidad de la información utilizada en la 
regresión. Según el Instituto Nacional de Estadística e Informática, la calidad de la información sobre los diferentes tipos de violencia en general y de los delitos contra el patrimonio en particular presenta limitaciones, debido -en parte- a la baja tasa de denuncias efectuadas por parte de las víctimas. A ello se adiciona la carencia de un registro estadístico anual de los diferentes tipos de violencia por departamentos para un período largo de tiempo.

Por tanto, las investigaciones posteriores de carácter departamental sobre el tema deberían tratar de cubrir dicho vacío, además de incorporar otros tipos de violencia a las consideradas en el presente estudio.

En el Perú el problema educativo radica más en el aspecto de la calidad que en la cantidad de años de estudio. Por ello, las regresiones deberían utilizar indicadores referidos a la calidad.

Dado que en la regresión se trabajó con valores promedio de 1994-2001 y 20022014, con la finalidad de darle una mayor consistencia a los parámetros estimados, se debería trabajar con información de panel, ello también en la medida que se disponga de la serie de datos para todas las variables consideradas y para todos los años del período de estudio.

Los resultados hallados, en tanto que indican que el capital humano y la integración comercial tienen una mayor influencia sobre el crecimiento económico departamental, sugiere que las autoridades departamentales deberían brindarle la prioridad necesaria a las políticas educativas y de inserción comercial al mercado internacional.

\section{BIBLIOGRAFÍA}

ADEX-Asociación de exportadores (2015). Boletín de Exportaciones Regionales - Adex Data Trade. Lima, Perú. (varios años).

Armendáriz, Edna; Jaramillo, Fidel y Zegarra, Luis (2011). Las barreras al crecimiento económico en Junín. BID y CIES: Lima, Perú, 126p.

Barro, Robert (1991). Economic growth in a cross section of countries. En: The Quarterly Journal of Economics, Vol. 106, No. 2, mayo, p. 407-443.

Barro, Robert (1996). Determinants of economic growth: a cross-country empirical study. NBER Working Paper 5698, Cambridge, 115p.

Barro, Robert y Sala-i-Martín, Xavier (1992). Convergence. En: Journal of Political Economy, Vol. 100, No. 2, abril, p. 223-251.

Becker, Gary (1993). Human capital: A theoretical and empirical analysis with special reference to education. The University of Chicago Press: Chicago, 376p. 
Bernal, Manuel y Castillo, Ramón (2012). Efecto de la delincuencia sobre la inversión extranjera directa en México. En: Revista Comercio Exterior, Vol. 62, No. 3, mayo-junio, p. 18-27.

Cotte, Alexander (2006). Crecimiento, desigualdad y pobreza: un análisis de la violencia en Colombia. En: Revista de Investigación, Vol. 6, No. 2, julio-diciembre, p. 209-222.

Glaeser, Edward; La Porta, Rafael; López-de-Silanes y Shleifer, Andrei (2004). Do institutions cause growth?. En: Journal of Economic Growth, Vol. 9, No. 3, setiembre, p. 271-303.

González, Salvador (2014). Criminalidad y crecimiento económico regional en México. En: Frontera Norte, Vol. 26, No. 51, enero-junio, p. 75-111.

Hofstetter, Marcel (1998). La violencia en los modelos de crecimiento económico. En: Revista de Economía de la Universidad del Rosario, Vol. 1, No. 2, noviembre, p. 67-77.

INEI-Instituto Nacional de Estadística e Informática (2003). Producto Bruto Interno por Departamentos 1994-2001. Cuentas Nacionales del Perú. INEI: Lima, Perú, 539p.

INEI-Instituto Nacional de Estadística e Informática (2013). Producto Bruto Interno por departamentos 2001-2012. Lima, Perú, 607p.

INEI-Instituto Nacional de Estadística e Informática (2014). Perú: Indicadores de educación por departamentos, 2001-2012. Lima, Perú, 325p.

INEI-Instituto Nacional de Estadística e Informática (2015). Producto Bruto Interno por departamentos 2014. Lima, Perú, 31p.

INEI-Instituto Nacional de Estadística e Informática (2015a). Compendio Estadístico del Perú. INEI: Lima, Perú. (De 1995 al 2015).

León, Juan (2005). Institucionalidad y crecimiento económico en el Perú. En: Revista Pensamiento Crítico, Vol. 4, No. 4, enero-junio, p. 2-22.

Lucas, Robert (1988). On the mechanics of economic development. En: Journal of Monetary Economics, Vol. 22, No. 1, julio, p. 3-42.

McMahon, Walter (2000). The impact of human capital on non-market outcomes and feedbacks on economic development, OECD, 47p.

Mendoza, Waldo y Gallardo, José (2011). Las barreras al crecimiento económico en Cajamarca. JICA: Lima, Perú, 123p.

Mankiw, Gregory; Romer, David y Weil, David (1992). A contribution to the empirics of economic growth. En: Quarterly Journal of Economics, Vol. 107, No. 2, mayo, p. 407-437.

North, Douglass (1993). Instituciones, cambio institucional y desempeño económico. Fondo de Cultura Económica: México, 220p.

Parra, Clara (1998). Determinantes de la inversión en Colombia: evidencia sobre el capital humano y la violencia. En: Archivos de macroeconomía No. 84: Bogotá, Colombia, 33p.

Ponce, Stefahnie (2013). Inversión pública y desarrollo económico regional. Tesis para optar el grado de Magister en Economía. Escuela de Postgrado, Pontificia Universidad Católica del Perú, Perú, 90p. 
Querubín, Pablo (2003). Crecimiento departamental y violencia criminal en Colombia. Documento CEDE 2003-12: Bogotá, Colombia, 49p.

Robles, Gustavo; Calderón, Gabriela y Magaloni, Beatriz (2013). Las consecuencias económicas de la violencia del narcotráfico en México. Documento de Trabajo del Banco Interamericano de Desarrollo, No. 426, 49p.

Rodrick, Dani; Subramanian, Arvind y Trebbi, Francesco (2002). Institutions rule: the primacy of institutions over geography and integration in economic development. NBER Working Paper Series 9305: Cambrigde, 44p.

Romer, Paul (1989). Increasing returns and new developments in the theory of growth. NBER Working Paper Series 3098: Cambrigde, 37p.

Romer, Paul (1994). The origins of endogenous growth. En: The Journal of Economic Perspective, Vol. 8, No. 1, invierno, p. 3-22.

Rubio, Mauricio (1995). Crimen y crecimiento en Colombia. En: Coyuntura Económica, Vol. 25, No.1, marzo, p. 101-125.

Solow, Robert (1957).Technical change and the aggregate production function. En: The Review of Economics and Statistics, Vol. 39, No. 3, agosto, p. 312-320.

Thirlwall, Antony (2003). La naturaleza del crecimiento económico: un marco alternativo para comprender el desempeño de las naciones. Fondo de Cultura Económica: México, 128p.

Tudela, Patricio y López, Beatriz (2005). Propuesta de marco conceptual para el estudio de políticas públicas de seguridad ciudadana. Los casos de Argentina, Chile y Uruguay (RPG-P1065). Banco Interamericano de Desarrollo: Washington, 22p.

Webb, Richard; Mendieta, Claudia y Agreda, Víctor (2012). Las barreras al crecimiento económico en Apurímac. BID y USMP: Lima, Perú, 181p. 